\title{
Composição florística de uma comunidade savânica no Rio Grande do Norte, Nordeste do Brasil
}

\author{
Ana Cláudia Pereira de Oliveira ${ }^{1}$, Alessandra dos Santos Penha ${ }^{2}$, \\ Raquel Franco de Souza ${ }^{3}$ e Maria Iracema Bezerra Loiola ${ }^{4,5}$
}

Recebido em 24/06/2011. Aceito em 26/04/2012

\begin{abstract}
RESUMO
(Composição florística de uma comunidade savânica no Rio Grande do Norte, Nordeste do Brasil). No município de Rio do Fogo foi registrada, em 1976, somente através de imagens de radar, uma comunidade de Cerrado para a qual, até então, não havia estudo in loco. Algumas questões foram elaboradas sobre essa comunidade: A região delimitada e descrita por meio de imagens de radar pode ser considerada Cerrado, do ponto de vista florístico? Qual a fitogeografia das espécies registradas nessa comunidade? Há ocorrência de espécies raras, endêmicas ou ameaçadas de extinção na composição florística dessa comunidade? Para responder a estas questões realizou-se o levantamento florístico em $400 \mathrm{ha}$, no período de agosto/2007 a setembro/2009. Os dados indicaram que das 94 espécies registradas em Rio do Fogo, 73 são associadas ao Cerrado, 69 também se distribuem na Caatinga, 60 na Floresta Atlântica, 47 na Floresta Amazônica, 14 no Pantanal e 11 no Pampa. Foram listadas pela primeira vez para o Rio Grande do Norte (RN) 39 espécies; Stylosanthes gracilis Kunth (Fabaceae) e Aristida laevis (Nees) Kunth (Poaceae) são citadas pela primeira vez para o Nordeste do Brasil. Aspilia procumbens Baker (Asteraceae) apresentou distribuição restrita ao estado e insere-se na categoria criticamente em perigo; já Stilpnopappus cearensis Hubber na categoria vulnerável à extinção. Concluiu-se que essa área pode ser classificada floristicamente como pertencente ao domínio do Cerrado, podendo ser apontada como área-chave para a conservação.
\end{abstract}

Palavras-chave: áreas-chave, biodiversidade, conservação, savana

\begin{abstract}
(Floristic composition of a savanna community in Rio Grande do Norte, northeastern Brazil). In the municipality of Rio do Fogo (in 1976) a savanna community was registered by radar that, until now, has not been studied in loco. Some questions were asked about this community: Is this area Cerrado, floristically? What is the phytogeography of plant species in this community? Do rare, endemic or endangered species occur in this community? To answer these questions a floristic inventory, from August 2007 to September 2009, was performed in 400 ha of this region. The data indicated that of the 94 species recorded, 73 are associated with Cerrado, 69 also with Caatinga, 60 with Atlantic Forest, 47 with Amazonian forest, 14 with the Pantanal and 11 with the Pampa flora. Thirty-nine species are first listed for Rio Grande do Norte (RN); Stylosanthes gracilis Kunth (Fabaceae) and Aristida laevis (Nees) Kunth (Poaceae) are first records for the Northeast of Brazil. Aspilia procumbens Baker (Asteraceae) showed a restricted distribution in the state and is listed as critically endangered. Stilpnopappus cearensis Hobber is listed as vulnerable to extinction. It was concluded that this area can be floristically classified as Cerrado, which points to a key area for conservation.
\end{abstract}

Key words: key areas, biodiversity, conservation, savanna

\footnotetext{
${ }^{1}$ Universidade Federal do Rio Grande do Norte, Programa de Pós-Graduação em Desenvolvimento e Meio Ambiente, Natal, RN, Brasil

${ }^{2}$ Universidade Federal de São Carlos, Departamento de Biotecnologia e Produção Vegetal e Animal, Campus Araras, São Carlos, SP, Brasil

${ }^{3}$ Universidade Federal do Rio Grande do Norte, Departamento de Geologia, Natal, RN, Brasil

${ }^{4}$ Universidade Federal do Ceará, Departamento de Biologia, Fortaleza, CE, Brasil

${ }^{5}$ Autor para correspondência: iloiola@yahoo.com.br
} 


\section{Introdução}

O Brasil apresenta a maior extensão de florestas tropicais do planeta e é intitulado como o país mais rico em biodiversidade, ficando em primeiro lugar em número de espécies de plantas (Barbosa \& Peixoto 2003; Ribeiro \& Walter 1998). Estima-se que a flora brasileira está representada por 42.903 espécies (Forzza et al. 2012).

Todavia, este número é certamente aproximado devido à escassez de estudos florísticos e taxonômicos, tanto em escala nacional quanto regional e local. Tomando como exemplo, temos a flora do Nordeste do Brasil que é estimada com cerca de 20.000 espécies de plantas (Barbosa et al. 1996). Segundo Tabarelli \& Vicente (2004), cerca de $40 \%$ da Caatinga, bioma predominante na região, nunca foi coletado e, do que já foi coletado $80 \%$ é subamostrado.

Apesar dessa lacuna de informações sobre a flora brasileira, uma investigação conduzida por Giulietti et al. (2009) que teve como objetivo identificar e mapear as espécies raras de plantas do Brasil e também as áreas mais importantes para a conservação dessas espécies, indicou para o país 2.256 espécies raras e 752 áreas-chave para a biodiversidade (ACBs) distribuídas da seguinte forma nas diferentes regiões: Sudeste 1.058 e $228\left(927.286 \mathrm{~km}^{2}\right)$; Nordeste 565 e $197\left(1.558 .200 \mathrm{~km}^{2}\right)$; Centro-Oeste 273 e $99\left(1.612 .088 \mathrm{~km}^{2}\right)$; Norte 235 e $154\left(3.869 .638 \mathrm{~km}^{2}\right)$ e Sul, 125 e $74\left(577.214 \mathrm{~km}^{2}\right)$.

Salienta-se que no estudo de Giulietti et al. (2009) o estado brasileiro com a maior representatividade em número de espécies raras foi Minas Gerais (550) e em número de ACBs a Bahia (152). Por outro lado, o Rio Grande do Norte foi o único estado para o qual não foi indicada nenhuma espécie rara e, consequentemente, nenhuma ACBs.

Neste contexto é notável a escassez de estudos botânicos, ecológicos, biogeográficos e fitossociológicos para o Rio Grande do Norte. Porém, o estado apresenta a Caatinga, como o domínio fitoecológico dominante, além de Floresta Atlântica e os ecossistemas associados, dentre estes as florestas ombrófilas densas e abertas, as florestas estacionais semideciduais e deciduais (Cestaro \& Soares 2008). Destaca-se aqui uma comunidade de Cerrado encravada entre a Caatinga e a Floresta Atlântica, mais precisamente no ecossistema associado às restingas entre os municípios de Rio do Fogo e Touros, conhecida até o momento somente por imagens de radar (RADAMBRASIL 1976).

Todavia, a principal área de distribuição do Cerrado concentra-se na região do Planalto Central, abrangendo os estados de Goiás, Tocantins, o Distrito Federal, porção leste do Mato Grosso e do Mato Grosso do Sul, porção oeste da Bahia e Minas Gerais (Eiten 1972; Eiten 1994; Mantovani \& Martins 1993). É encontrado também em áreas marginais, como nos tabuleiros nordestinos no Maranhão, Piauí e Ceará, assim como no Amazonas e em áreas disjuntas localizadas nos limites meridionais do domínio fitoecológico, nos estados de São Paulo e Paraná (Eiten 1994; Ratter et al. 1997; Vargas \& Hungria 1997).
Diante do exposto e também por não haver estudos in loco nessa comunidade, algumas questões foram elaboradas: 1) A região delimitada e descrita por meio de imagens de radar pode ser considerada Cerrado, do ponto de vista florístico? 2) Qual a fitogeografia das espécies registradas na área? 3) Há ocorrência de espécies raras, endêmicas ou ameaçadas de extinção?

O objetivo desse estudo foi confirmar in loco a existência florística do Cerrado no Rio Grande do Norte, bem como avaliar a importância da flora vascular do Rio Grande do Norte, visando fornecer dados para que o estado possa ser contemplado com ACBs.

\section{Material e métodos}

\section{Área de estudo}

Abrangendo cerca de 400 hectares, está localizada no município do Rio do Fogo (05²4'948' S e 35²3’015” W), microrregião Nordeste do estado do Rio Grande do Norte (Figs.1 e 2). Até 1978, esta área pertencia ao Estado e foi doada à União Federal que, em 2004, deu a certidão de posse ao $17^{\circ}$ Grupo de Artilharia de Campanha (GAC) do Exército Brasileiro.

De acordo com Köppen (1948), o clima é do tipo As' (clima tropical). A pluviosidade média anual varia entre 800 a $1.200 \mathrm{~mm}$ e o solo é classificado como Areias Quartzosas Marinhas (Neossolos Quartzarênicos Marinhos), representado por formações do Grupo Barreiras (Andrade 1967; IDEMA 2002).

\section{Levantamento florístico}

A amostragem de material botânico foi realizada no período de agosto/2007 a setembro/2009, por meio de excursões mensais com duração média de cinco dias, nos quais foram realizadas caminhadas assistemáticas por toda a área. No campo, foram anotados dados referentes à localização da espécie, hábito, altura (no caso de arbóreas, estimados usando-se como comparação a vara de coleta de tamanho conhecido), presença de látex e cor das flores e/ou frutos. Cada local de coleta foi devidamente referenciado utilizando um GPS (Global Positioning System) modelo Legend HCx.

As amostras foram herborizadas conforme os procedimentos usuais em trabalhos de levantamentos florísticos (Mori et al., 1989) e identificadas com o auxilio de especialistas, chaves de identificações e/ou por comparação com coleções dos Herbários UFRN, ESA, IBGE e UB. O material foi depositado no Herbário UFRN e as duplicatas doadas aos herbários ESA, TEPB e EAC, cujos acrônimos encontram-se listados conforme Thiers (2012).

A circunscrição das famílias seguiu APG III (2009) e os nomes dos autores das espécies foram aferidos nas bases de dados disponíveis na internet (Missouri 2008) e com o apoio do aplicativo Plantminer (Carvalho et al. 2010). 


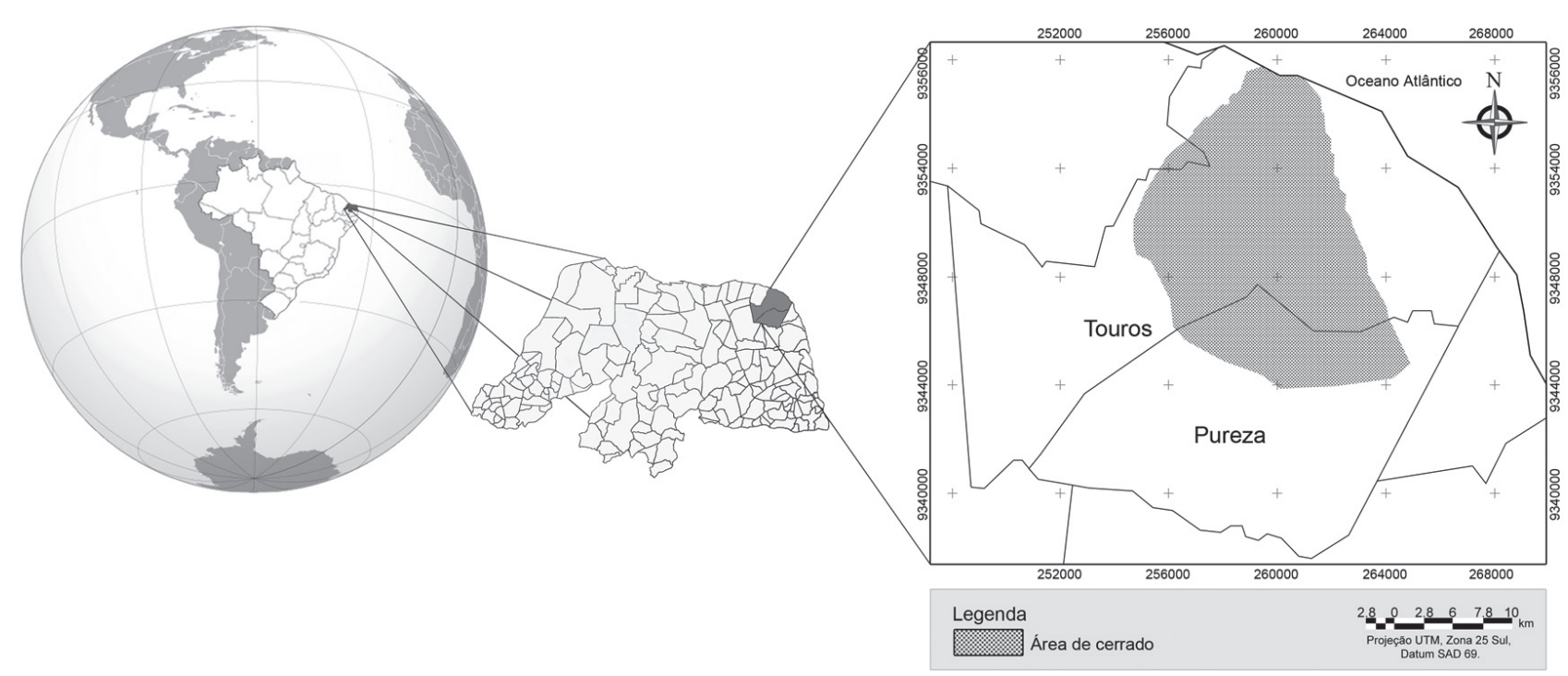

Figura 1. Localização da área de estudo no município de Rio do Fogo, Rio Grande do Norte, Brasil.

\section{Distribuição fitogeográfica}

A distribuição das espécies foi baseada em Forzza et al. (2012).

\section{Novos registros, espécies raras, endêmicas e ameaçadas}

Para esta classificação foram utilizadas as seguintes bibliografias: i) "novos registros" - foram consultadas diferentes obras na literatura e a "Lista de espécies da flora do Brasil" (Forzza et al. 2012);

ii) "rara" - foram consultados o livro "Plantas Raras do Brasil" (Giulietti et al., 2009), bem como a "Lista de espécies da flora do Brasil" (Forzza et al. 2012);

iii) "endêmica" - consultou-se a "Lista de espécies da flora do Brasil" (Forzza et al. 2012);

iv) ameaçada" - para este fim, utilizou-se a "Lista oficial do Instituto Brasileiro do Meio Ambiente e dos Recursos Naturais Renováveis" (MMA, 2008), a "Lista Vermelha” da União Internacional para a Conservação da Natureza (2001) e a Lista da Biodiversitas (2008).

\section{Resultados}

\section{Levantamento Florístico}

Foram registradas na área de estudo 94 espécies pertencentes a 74 gêneros e representando 35 famílias de plantas vasculares (Tab. 1). As famílias com maior riqueza de espécies foram Fabaceae (15), seguida de Poaceae (13), Convolvulaceae e Rubiaceae (com seis espécies cada), sendo que em 23 famílias ( $25 \%$ ) ocorreram até duas espécies. As famílias de maior riqueza de gêneros foram Fabaceae e Poaceae (10 cada), seguida de Rubiaceae (cinco), Convol- vulaceae e Euphorbiaceae (quatro cada). Aproximadamente $26 \%$ (24) das famílias foram representadas por um único gênero. Os gêneros com maior riqueza de espécies foram Bulbostylis (Cyperaceae) e Stylosanthes (Fabaceae) com quatro espécies, Axonopus (Poaceae) e Hirtella (Chrysobalanaceae) com três, sendo que 12 gêneros ( $13 \%$ do total) foram representados por duas espécies e 55 gêneros (59\%) por uma única espécie.

Verificou-se o predomínio do hábito herbáceo ou subarbustivo, estando representado por 54 espécies (58 \%) em 22 famílias, seguido do hábito arbustivo ou arbóreo, registrado em 31 espécies (33\%) e 15 famílias. No componente herbáceo ou subarbustivo destacaram-se as famílias Poaceae (15 espécies), Fabaceae (nove), Cyperaceae (quatro), Rubiaceae e Convolvulaceae (três cada), e no componente arbustivo ou arbóreo Fabaceae (cinco), Myrtaceae (quatro), Chrysobalanaceae e Rubiaceae (três).

\section{Distribuição fitogeográfica}

Das 94 espécies registradas no presente estudo, 73 (77,6\%) foram citadas na "Lista de Espécies da Flora do Brasil" (Forzza et al. 2012), como associadas ao Cerrado. Ainda, segundo esses autores, $69(73,4 \%)$ das espécies também se distribuem na Caatinga, 60 (63,8 \%) na Floresta Atlântica, 47 (50 \%) na Floresta Amazônica, 14 (14,9 \%) no Pantanal e $11(11,7 \%)$ no Pampa (Tab.1).

No contexto da distribuição fitogeográfica, as espécies que se destacaram com ocorrências nos seis domínios considerados por Forzza et al. (2012), foram: Anacardium occidentale L. (Anacardiaceae), Polycarpaea corymbosa (L.) Lam. (Caryophyllaceae), Bulbostylis junciformis (Humb., Bonpl. \& Kunth) C.B.Clarke (Cyperaceae) e Casearia sylvestris Sw. (Salicaceae). 


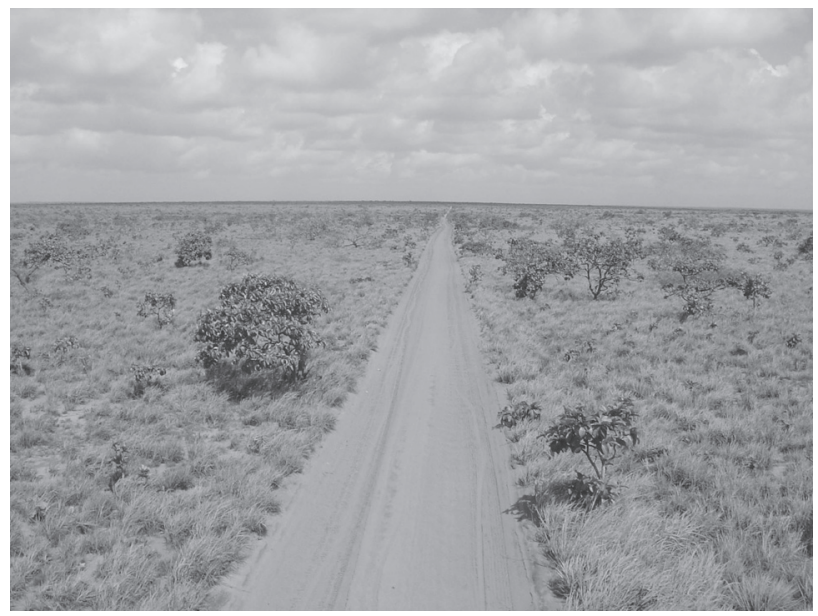

Figura 2. Vista geral da área estudada em Rio do Fogo, Rio Grande do Norte, Brasil.

\section{Novos registros, espécies ameaçadas e endêmicas}

De acordo com a Lista das Espécies da Flora do Brasil (Forzza et al. (2012) das 94 espécies listadas na área de estudo, $40(42,5 \%)$ foram registradas pela primeira vez no estado do Rio Grande do Norte (Tab. 1). Aristida laevis (Nees) Kunth (Poaceae) é citada pela primeira vez para o Nordeste do Brasil.

Foi registrada somente uma espécie rara para a comunidade estudada, Aspilia procumbens Baker (Asteraceae) que também foi inserida pela Biodiversitas (2008) na categoria criticamente em perigo.

Sobre o endemismo, foi possível observar que 24 (25,5\%) espécies registradas em Rio do Fogo têm distribuição restrita ao território brasileiro (Forzza et al. 2012).

\section{Discussão}

\section{Levantamento Florístico}

As famílias de maior riqueza de espécies Fabaceae, Poaceae, Convolvulaceae e Rubiaceae registradas neste trabalho, também são apontadas em outros estudos desenvolvidos nos cerrados do Centro-Oeste (Eiten 1972; Filgueiras \& Pereira 1990); além disso, essas famílias são consideradas comuns no Cerrado por alguns autores (Eiten 1972; Mantovani \& Martins 1993; Mendonça et al. 1998; Filgueiras 2002; Andrade et al. 2002; Haidar et al. 2005).

A maior diversidade de Fabaceae, possivelmente, está associada a sua ampla distribuição no Cerrado, ocorrendo desde campo limpo a formações florestais (Durigan et al. 1997; Costa \& Araújo 2001; Balduino et al. 2005). Esta família destacou-se também em outros estudos tanto no estrato subarbustivo ou herbáceo (Filgueiras \& Pereira 1990; Batalha \& Mantovani 2000; Costa et al. 2004) quanto no estrato arbustivo ou arbóreo (Felfili \& Silva Jr. 1993; Batalha \& Mantovani 2000; Costa \& Araújo 2001; Weiser \& Godoy 2001; Costa et al. 2004). Merece destacar que, o sucesso deste grupo taxonômico está, provavelmente, relacionado à presença de nódulos nas raízes, que funcionam como um mecanismo de retenção e transferência de nutrientes. Desta forma, considera-se que os representantes de Fabaceae são extremamente importantes tanto na estrutura da vegetação do Cerrado, como em outros domínios fitogeográficos, sendo essencial para a dinâmica dessas comunidades (Martins 1993).

O sucesso ecológico das Poaceae, não só no Cerrado, mas também em diferentes tipos de hábitats, incluindo a região da Antártida (Kellogg 1998), deve-se principalmente à diversidade genética da família, o que resultou em adaptações morfológicas a ambientes diversos. Entre essas adaptações, podemos citar a tolerância à dessecação e a capacidade de crescer em lugares secos e abertos (Kellogg 2001). Os gêneros Andropogon L., Axonopus P. Beauv. e Trachypogon Nees, registrados na área de estudo também foram apontados como componentes importantes em outros levantamentos florísticos e fitossociológicos do Cerrado (Goodland 1979; Mantovani \& Martins 1993; Felfili et al. 1994; Silva \& Nogueira 1999; Munhoz \& Felfili 2006). De acordo com Filgueiras et al. (1998), os representantes de Poaceae podem corresponder até $25 \%$ do número total de espécies do Cerrado da região central.

Outros gêneros frequentemente associados ao domínio dos Cerrados e que também foram registrados na área de estudo são: Byrsonima Rich. ex Kunth (Goodland 1979), Eugenia L., Myrcia DC., Anacardium L., Erythroxylum Kunth e Tocoyena Aubl. (Ratter et al. 1997; Rizzini 1997).

\section{Distribuição fitogeográfica}

Apesar da maioria das espécies $(77,6 \%)$ registradas em Rio do Fogo estar associada ao Cerrado, um número muito expressivo também pode ocorrer na Caatinga $(73,4 \%)$ e na Restinga $(63,8 \%)$ e tal fato pode, provavelmente, ser decorrente do posicionamento geográfico da área estudada. Esta se encontra encravada entre a Caatinga e a Restinga e, possivelmente, recebe influência florística das mesmas. A justaposição ou interpenetração de espécies entre fitofisionomias foi constatada também em estudos realizados no sudeste brasileiro (Pereira \& Araújo 2000; Cerqueira 2000; Méio et al. 2003; Scarano et al. 2004). Cabe ainda destacar o estudo realizado por Rizzini (1963) no estado de São Paulo, que concluiu que mais da metade da flora do componente do Cerrado provém da Floresta Atlântica, dependendo da localização da área em estudo.

Desta forma, não se deve desprezar o fato da amplitude geográfica dessas espécies. Cada espécie tem sua própria e única distribuição e algumas destas distribuições indicam limites impostos pelo presente ambiente e outras refletem conexões e climas passados (Brow \& Gibson 1983). Tomando-se como exemplo as espécies Polycarpaea corymbosa, Bulbostylis junciformis e Casearia sylvestris que segundo Forzza et al. (2012) são generalistas, estas podem ocorrem em todos os domínios fitogeográficos brasileiros. Destaca-se 
Tabela 1. Lista de espécies de Angiospermas registradas em Rio do Fogo, RN - Brasil com as respectivas distribuição geográfica e nos domínios fitogeográficos de acordo com Forzza et al. (2012). Espécies em negrito = primeira ocorrência para o RN, A= primeiro registro para o Nordeste do Brasil, B = ocorrem apenas no Nordeste, $\mathrm{C}=$ restrita ao RN. Domínios fitogeográficos: AM = Amazônia; CAA= Caatinga; $\mathrm{CE}=$ Cerrado; $\mathrm{MA}=$ Mata Atlântica; PAM= Pampa; PAT= Pantanal. Estados brasileiros: $\mathrm{AC}=\mathrm{Acre} ; \mathrm{AL}=$ Alagoas; $\mathrm{AM}=$ Amazonas; $\mathrm{AP}=$ Amapá; $\mathrm{BA}=\mathrm{Bahia} ; \mathrm{CE}=$ Ceará; $\mathrm{ES}=$ Espírito Santo; $\mathrm{GO}=\mathrm{Goiás} ; \mathrm{MA}=\mathrm{Maranhão} ; \mathrm{MT}=$ Mato Grosso; MS = Mato Grosso do Sul; MG = Minas Gerais; PA = Pará; PB = Paraíba; PR = Paraná; PE = Pernambuco; PI = Piauí; RJ = Rio de Janeiro; RN = Rio Grande do Norte; $\mathrm{RS}=$ Rio Grande do Sul; $\mathrm{RO}=$ Rondônia; $\mathrm{RR}=$ Roraima; $\mathrm{SC}=$ Santa Catarina; $\mathrm{SE}=$ Sergipe; $\mathrm{SP}=$ São Paulo; $\mathrm{TO}=\mathrm{Tocantins} ; \mathrm{DF}=\mathrm{Distrito}$ Federal .

\begin{tabular}{|c|c|c|c|}
\hline FAMÍLIA & ESPÉCIES & $\begin{array}{l}\text { DOMÍNIOS } \\
\text { FITOGEOGRÁFICOS } \\
\text { (Forzza et al., 2012) }\end{array}$ & $\begin{array}{l}\text { DISTRIBUIÇÃO GEOGRÁFICA } \\
\text { (Forzza et al., 2012) }\end{array}$ \\
\hline ANACARDIACEAE & Anacardium occidentale $\mathrm{L}$. & AM, CAA, CE, MA, PAM, PAT & $\begin{array}{l}\text { Norte (RR, AP, AM, TO, AC), Nordeste, } \\
\text { Centro-Oeste (MT, GO, DF), Sudeste, Sul (PR, SC) }\end{array}$ \\
\hline \multirow[t]{3}{*}{ APOCYNACEAE } & Ditassa crassifolia Decne & CE, MA, & $\begin{array}{l}\text { Nordeste (RN, PB, PE, BA, AL, SE), } \\
\text { Sudeste (ES) }\end{array}$ \\
\hline & Hancornia speciosa Gomes & $\mathrm{AM}, \mathrm{CAA}, \mathrm{CE}$ & $\begin{array}{l}\text { Norte (AP, PA, AM, TO, RO), } \\
\text { Nordeste (MA, PI, RN, PB, PE, BA, AL, SE), } \\
\text { Centro-Oeste, Sudeste, Sul (PR) }\end{array}$ \\
\hline & $\begin{array}{l}\text { Mandevilla scabra (Hoffmanns. } \\
\text { ex Roem. \& Schult.) K.Schum }\end{array}$ & $\mathrm{AM}, \mathrm{CAA}, \mathrm{CE}, \mathrm{MA}$ & $\begin{array}{l}\text { Norte (RR, PA, AM, AC, RO), } \\
\text { Nordeste (PI, CE, RN, PB, PE, BA, AL, SE), } \\
\text { Centro-Oeste (MT, GO, DF), Sudeste, Sul }\end{array}$ \\
\hline \multirow[t]{2}{*}{ ASTERACEAE } & ${ }_{B, C}$ Aspilia procumbens Baker & CAA & Nordeste (RN) \\
\hline & ${ }^{B}$ Stilpnopappus cearensis Hubber & CAA & Nordeste (CE, RN, PB, PE) \\
\hline CARYOPHYLACEAE & $\begin{array}{l}\text { Polycarpaea corymbosa } \\
\text { (L.) Lam. }\end{array}$ & AM, CAA, CE, MA, PAM, PAT & $\begin{array}{l}\text { Norte (RR, PA, TO, AC), Nordeste (PB, PE, BA), } \\
\text { Centro-Oeste (MG, GO, DF, MS), Sudeste (MG, SP), Sul (PR) }\end{array}$ \\
\hline CELASTRACEAE & Maytenus erythroxylon Reiss. & $\mathrm{CAA}, \mathrm{CE}$ & Centro-Oeste (MT) \\
\hline \multirow[t]{3}{*}{ CHRYSOBALANACEAE } & $\begin{array}{l}\text { Hirtella gracilipes } \\
\text { (Hook.f.) Prance }\end{array}$ & $\mathrm{AM}, \mathrm{CAA}, \mathrm{CE}$ & $\begin{array}{l}\text { Norte (PA, TO, RO), Nordeste (PI, BA), } \\
\text { Centro-Oeste (MT, GO, DF, MT), Sudeste (MG, SP, RJ) }\end{array}$ \\
\hline & Hirtella ciliata Mart. \& Zucc. & $\mathrm{AM}, \mathrm{CAA}, \mathrm{CE}$ & $\begin{array}{l}\text { Norte (AM, PA, TO), Nordeste (MA, PI, CE, PE, BA, SE), } \\
\text { Centro-Oeste (GO), Sudeste (MG) }\end{array}$ \\
\hline & Hirtella racemosa Lam. & AM, CAA, CE, MA & $\begin{array}{l}\text { Norte (RR, AM, PA, AM, AC, RO), Nordeste (MA, CE, PA, } \\
\text { PE, BA, SE), Centro-Oeste (MT, GO, DF, MS) }\end{array}$ \\
\hline \multirow[t]{6}{*}{ CONVOLVULACEAE } & $\begin{array}{l}\text { Evolvulus glomeratus } \\
\text { Nees \& C. Mart. }\end{array}$ & AM, CAA, CE, MA, PAM & $\begin{array}{c}\text { Norte (RR, PA, AM, AC), Nordeste (PI, CE, PB, PE, BA), } \\
\text { Centro-Oeste, Sudeste, Sul }\end{array}$ \\
\hline & Evolvulus lithospermoides Mart. & $\mathrm{CE}$ & $\begin{array}{l}\text { Nordeste (BA), Centro-Oeste (MG, GO), } \\
\text { Sudeste (MG, SP) }\end{array}$ \\
\hline & Ipomoea brasiliana Meisn. & CAA & Nordeste (MA, PI, CE, RN, PB, PE, BA, AL, SE) \\
\hline & $\begin{array}{l}\text { Jacquemontia menispermoides } \\
\text { Choisy }\end{array}$ & $\mathrm{CE}$ & Nordeste (PE, PB), Sudeste (RJ) \\
\hline & Jacquemontia serrata Meisn. & CE, MA & Nordeste (CE, RN, PB, PE, BA), Sudeste (RJ, MG) \\
\hline & $\begin{array}{l}\text { Merremia digitata (Spreng.) } \\
\text { Hallier } \mathrm{f} .\end{array}$ & CAA, CE & $\begin{array}{l}\text { Norte (TO), Nordeste (BA), } \\
\text { Centro-Oeste (MG, GO, DF, MS), } \\
\text { Sudeste (MG, SP), Sul (PR) }\end{array}$ \\
\hline \multirow[t]{5}{*}{ CYPERACEAE } & $\begin{array}{l}\text { Bulbostylis capillaris (L.) } \\
\text { C.B.Clarke }\end{array}$ & CAA, CE, MA, PAM & Nordeste, Centro-Oeste, Sudeste, Sul \\
\hline & $\begin{array}{l}\text { Bulgostylis conifera (Kunth) } \\
\text { C.B.Clarke }\end{array}$ & $\mathrm{AM}, \mathrm{CAA}, \mathrm{CE}, \mathrm{MA}$ & Norte, Nordeste, Centro-Oeste, Sudeste \\
\hline & $\begin{array}{l}\text { Bulbostylis junciformis } \\
\text { (Humb., Bonpl. \& Kunth) } \\
\text { C.B.Clarke }\end{array}$ & AM, CAA, CE, MA, PAM, PAT & Norte, Nordeste, Centro-Oeste, Sudeste, Sul \\
\hline & $\begin{array}{c}\text { Bulbostylis scabra } \\
\text { (J.Presl \& C.Presl) C.B.Clarke }\end{array}$ & BA, MG, MS, PB, PE, RS & Nordeste, Centro-Oeste, Sudeste, Sul \\
\hline & $\begin{array}{l}\text { Rhynchospora nervosa } \\
\text { (Vahl) Boeckeler }\end{array}$ & AM, CAA, CE, MA, PAT & Norte, Nordeste, Centro-Oeste, Sudeste, Sul \\
\hline
\end{tabular}


Tabela 1. Continuação.

\begin{tabular}{|c|c|c|c|}
\hline FAMÍLIA & ESPÉCIES & $\begin{array}{c}\text { DOMÍNIOS } \\
\text { FITOGEOGRÁFICOS } \\
\text { (Forzza et al., 2012) }\end{array}$ & $\begin{array}{l}\text { DISTRIBUIÇÃO GEOGRÁFICA } \\
\text { (Forzza et al., 2012) }\end{array}$ \\
\hline DILLENIACEAE & Curatella americana $\mathrm{L}$. & $\mathrm{AM}, \mathrm{CAA}, \mathrm{CE}, \mathrm{MA}$ & $\begin{array}{l}\text { Norte (RR, AP, PA, AM, TO, RO), } \\
\text { Nordeste, Centro-Oeste, Sudeste (MG) }\end{array}$ \\
\hline ERYTHROXYLACEAE & $\begin{array}{l}\text { Erythroxylum suberosum var. } \\
\text { denudatum O.E.Schulz }\end{array}$ & $\mathrm{AM}, \mathrm{CAA}, \mathrm{CE}$ & $\begin{array}{l}\text { Norte (RR, AP, PA, AM, TO, RO), Nordeste (MA, PI, CE, } \\
\quad \text { PB, PE, BA, AL), Centro-Oeste, Sudeste, Sul (PR) }\end{array}$ \\
\hline \multirow[t]{4}{*}{ EUPHORBIACEAE } & $\begin{array}{l}\text { Chamaesyce prostrata } \\
\text { (Aiton) Small }\end{array}$ & CAA, CE, MA & $\begin{array}{c}\text { Norte (PA, AM, AP), Nordeste (CE, RN, PB, PE, SE, BA), } \\
\text { Centro-Oeste (MT), Sudeste, Sul (PR, SC) }\end{array}$ \\
\hline & Croton adamantinus Müll. Arg. & CAA & Nordeste (PI, CE, RN, PE, BA, SE), Sudeste (MG) \\
\hline & Manihot glaziovii Müll. Arg. & CAA & Sem Informações \\
\hline & $\begin{array}{l}\text { Pera glabrata (Schott) } \\
\quad \text { Poepp. ex Baill. }\end{array}$ & AM, CAA, CE, MA & $\begin{array}{l}\text { Norte (AP, PA, AM, AC, RO), Nordeste (MA, CE, PB, PE, } \\
\text { BA), Centro-Oeste (MT, GO, DF), Sudeste, Sul }\end{array}$ \\
\hline \multirow[t]{16}{*}{ FABACEAE } & $\begin{array}{l}\text { Sebastiania corniculata (Vahl) } \\
\text { Müll. Arg. }\end{array}$ & MA & Sem Informações \\
\hline & $\begin{array}{l}\text { Chamaecrista flexuosa (L.) } \\
\text { Greene }\end{array}$ & AM, CAA, CE, MA, PAT & $\begin{array}{l}\text { Norte (RR, PA, AM, TO, RO), } \\
\text { Nordeste (MA, PI, CE, RN, PB, PE, BA, AL), } \\
\text { Centro-Oeste, Sudeste, Sul }\end{array}$ \\
\hline & $\begin{array}{l}\text { Chamaecrista ramosa }(\text { Vogel) } \\
\text { H.S.Irwin \& Barneby }\end{array}$ & AM, CAA, CE, MA, PAT & $\begin{array}{l}\text { Norte (RR, AM, PA, AM, TO), Nordeste, } \\
\quad \text { Centro-Oeste, Sudeste, Sul (PR) }\end{array}$ \\
\hline & $\begin{array}{l}\text { Senna macranthera (DC. ex } \\
\text { Collad.) H.S.Irwin \& Barneby }\end{array}$ & CAA, CE, MA & $\begin{array}{c}\text { Norte (TO), Nordeste (PI, CE, RN, PB, PE, BA, AL), } \\
\text { Centro-Oeste (MG, GO, DF), } \\
\text { Sudeste, Sul (PR, RS) }\end{array}$ \\
\hline & 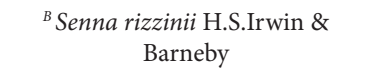 & $\mathrm{CAA}, \mathrm{CE}$ & Nordeste (CE, PB, BA), Centro-Oeste (MT) \\
\hline & Aeschynomene histrix Poir. & $\mathrm{AM}, \mathrm{CAA}, \mathrm{CE}$ & $\begin{array}{c}\text { Norte (RR, AP, PA, AM, AC), } \\
\text { Nordeste (PI, CE, PE, BA, AL), } \\
\text { Centro-Oeste (MT, GO, MS), } \\
\text { Sudeste (MG), Sul (PR) }\end{array}$ \\
\hline & Andira humilis Mart. ex Benth. & CAA, CE & $\begin{array}{l}\text { Norte (PA, RO), Nordeste (MA, RN, PE, BA), } \\
\text { Centro-Oeste (MG, DF, MS), Sudeste (MG, SP), Sul (PR) }\end{array}$ \\
\hline & $\begin{array}{c}\text { Centrosema brasilianum (L.) } \\
\text { Benth. }\end{array}$ & AM, CAA, CE, MA, PAT & $\begin{array}{l}\text { Norte (RR, AP, PA, AM), Nordeste, } \\
\text { Centro-Oeste, Sudeste, Sul (PR, SC) }\end{array}$ \\
\hline & $\begin{array}{l}\text { Desmodium barbatum (L.) } \\
\text { Benth. }\end{array}$ & AM, CAA, CE, MA, PAM, PAT & $\begin{array}{l}\text { Norte (PA, AC, RO), Nordeste (MA, CE, BA, SE), } \\
\text { Centro-Oeste, Sudeste, Sul }\end{array}$ \\
\hline & Stylosanthes angustifolia Vogel & $\mathrm{AM}, \mathrm{CAA}, \mathrm{CE}, \mathrm{MA}$ & $\begin{array}{l}\text { Norte (RR, PA, AM, AC, RO), } \\
\text { Nordeste (MA, PI, CE, RN, PB, PE, BA, SE) }\end{array}$ \\
\hline & Stylosanthes humilis Kunth & $\mathrm{AM}, \mathrm{CAA}, \mathrm{CE}, \mathrm{MA}$ & $\begin{array}{l}\text { Norte (PA, AM), Nordeste (MA, CE, PB, BA, AL), } \\
\quad \text { Centro-Oeste (GO, DF, MS), Sudeste (SP) }\end{array}$ \\
\hline & Stylosanthes scabra Vogel & $\mathrm{AM}, \mathrm{CAA}, \mathrm{CE}, \mathrm{MA}$ & $\begin{array}{l}\text { Norte (RR, PA), Nordeste (MA, PI, CE, RN, } \\
\text { PB, PE, BA, AL), Centro-Oeste, Sudeste }\end{array}$ \\
\hline & Stylosanthes gracilis Kunth & $\mathrm{AM}, \mathrm{CAA}, \mathrm{CE}, \mathrm{MA}$ & $\begin{array}{c}\text { Norte (AP, PA), Nordeste (MA, CE, PE, BA, AL, SE), } \\
\text { Centro-Oeste (MT, GO, DF, MS), } \\
\text { Sudeste (MG, ES, SP, RJ), Sul (PR) }\end{array}$ \\
\hline & Tephrosia purpurea (L.) Pers. & $\mathrm{AM}, \mathrm{CAA}, \mathrm{MA}$ & Nordeste, Sudeste (MG, SP, RJ) \\
\hline & ${ }^{B}$ Mimosa misera Benth. & $\mathrm{CAA}, \mathrm{CE}$ & Nordeste (PI, CE, BA) \\
\hline & $\begin{array}{l}\text { Ptyrocarpa moniliformis (Benth.) } \\
\text { Luckow \& R.W.Jobson }\end{array}$ & $\mathrm{CAA}, \mathrm{CE}$ & Nordeste (MA, PI, PB, PE, BA), Centro-Oeste (MT) \\
\hline KRAMERIACEAE & Krameria tomentosa A.St.-Hil. & AM, CAA, CE, MA & $\begin{array}{l}\text { Norte (PA, AM), Nordeste (MA, PI, CE, RN, PB, PE, BA), } \\
\text { Centro-Oeste (MT, GO), Sudeste (MG, RJ) }\end{array}$ \\
\hline
\end{tabular}


Tabela 1. Continuação.

\begin{tabular}{|c|c|c|c|}
\hline FAMÍLIA & ESPÉCIES & $\begin{array}{c}\text { DOMÍNIOS } \\
\text { FITOGEOGRÁFICOS } \\
\text { (Forzza et al., 2012) }\end{array}$ & $\begin{array}{l}\text { DISTRIBUIÇÃO GEOGRÁFICA } \\
\quad \text { (Forzza et al., 2012) }\end{array}$ \\
\hline \multirow[t]{2}{*}{ LAMIACEAE } & Aegiphila selowiana Cham. & MA & Sem informações \\
\hline & $\begin{array}{l}\text { Marsypianthes chamaedrys } \\
\text { (Vahl) Kuntze }\end{array}$ & AM, CAA, CE, MA, PAM & $\begin{array}{l}\text { Norte (PA, AM, TO, AC, RO), } \\
\text { Nordeste (MA, PI, CE, PB, PE, BA, AL, SE), } \\
\text { Centro-Oeste, Sudeste, Sul (PR) }\end{array}$ \\
\hline LAURACEAE & Cassytha americana Nees & $\mathrm{AM}, \mathrm{CAA}, \mathrm{CE}, \mathrm{MA}$ & $\begin{array}{l}\text { Norte (RR, PA, AM, RO), Nordeste (CE, RN, PB, BA), } \\
\text { Centro-Oeste (MS), Sudeste (SP, ES) }\end{array}$ \\
\hline LOGANIACEAE & Strychnos parvifolia A.DC. & $\mathrm{AM}, \mathrm{CAA}, \mathrm{CE}, \mathrm{MA}$ & $\begin{array}{l}\text { Norte (PA, AM, AC), } \\
\text { Nordeste (MA, PI, CE, RN, PB, PE, BA), Sudeste }\end{array}$ \\
\hline LORANTHACEAE & $\begin{array}{l}\text { Struthanthus flexicaulis } \\
\text { (Mart. ex Schult. f.) Mart. }\end{array}$ & CE, MA & $\begin{array}{l}\text { Nordeste (BA), Centro-Oeste (GO, DF), } \\
\text { Sudeste (MG, SP, RJ), Sul (PR, SC) }\end{array}$ \\
\hline LYTHRACEAE & Cuphea flava Spreng. & MA & Nordeste (PB, PE, BA, SE), Sudeste (ES, RJ) \\
\hline \multirow[t]{3}{*}{ MALPIGHIACEAE } & Byrsonima crassifolia (L.) Kunth & AM, CAA, CE & $\begin{array}{c}\text { Norte (RR, AP, PA, TO), Nordeste (MA, PI, PE, BA), } \\
\text { Centro-Oeste, Sudeste (MG, SP) }\end{array}$ \\
\hline & Byrsonima gardneriana A.Juss. & CAA, CE & Norte (AM), Nordeste (CE, RN, PB, PE, BA, AL) \\
\hline & Stigmaphyllon paralias A.Juss. & CAA, CE, MA & $\begin{array}{c}\text { Nordeste (MA, PI, CE, RN, PB, PE, BA, AL, SE), Centro- } \\
\text { Oeste (GO), Sudeste (ES, RJ) }\end{array}$ \\
\hline \multirow[t]{2}{*}{ MALVACEAE } & $\begin{array}{l}\text { Waltheria bracteosa A. St.-Hil. } \\
\text { \& Naudin }\end{array}$ & $\mathrm{AM}, \mathrm{CAA}, \mathrm{CE}, \mathrm{MA}$ & $\begin{array}{c}\text { Norte (TO), Nordeste (MA, PI, BA), } \\
\text { Centro-Oeste (MT, GO, MS), Sudeste (MG) }\end{array}$ \\
\hline & Waltheria indica $\mathrm{L}$. & AM, CAA, MA & $\begin{array}{l}\text { Norte (AM, PA, AM, TO, AC), Nordeste (CE, PB, PE, BA, } \\
\text { AL, SE), Centro-Oeste, Sudeste, Sul (PR, SC) }\end{array}$ \\
\hline \multirow[t]{5}{*}{ MYRTACEAE } & $\begin{array}{c}\text { Campomanesia guaviroba (DC.) } \\
\text { Kiaersk }\end{array}$ & CE, MA & Nordeste (BA), Sudeste (MG, ES, SP), Sul \\
\hline & $\begin{array}{l}\text { Eugenia luschnathiana (O.Berg) } \\
\text { Klotzsch ex B.D.Jacks. }\end{array}$ & Dados não obtidos & Nordeste (Ceará, Bahia) \\
\hline & Eugenia punicifolia (Kunth) DC. & $\mathrm{CE}$ & $\begin{array}{l}\text { Norte (RR, AP, PA, AM, AC, RO), Nordeste (MA, CE, RN, PE, } \\
\text { BA), Centro-Oeste (MT, GO, DF, MS), Sudeste (MG, SP, RJ) }\end{array}$ \\
\hline & Myrcia guianensis (Aublet.) DC. & $\mathrm{AM}, \mathrm{CAA}, \mathrm{CE}, \mathrm{MA}$ & $\begin{array}{c}\text { Norte (AP, PA, AM, AC), } \\
\text { Nordeste (CE, RN, PE, BA, AL), } \\
\text { Centro-Oeste (MT, GO, MS), Sudeste, Sul (SC, RS) }\end{array}$ \\
\hline & Myrciaria tenella (DC.) O.Berg. & $\mathrm{CE}$ & $\begin{array}{l}\text { Norte (PA, AM), Nordeste (MA), } \\
\text { Sudeste (SP, RJ), Sul (PR, RS) }\end{array}$ \\
\hline NYCTAGINACEAE & Pisonia cordifolia Mart. & MA & Dados não obtidos \\
\hline OCHNACEAE & Ouratea castaneifolia (DC.) Engl. & CAA, CE & $\begin{array}{l}\text { Norte (PA, AM, TO), Nordeste (MA, PI, BA), } \\
\text { Centro-Oeste (MT, GO, DF), Sudeste (MG, RJ) }\end{array}$ \\
\hline ORCHIDACEAE & Cyrtopodium punctatum (L.) Lindl. & $\mathrm{CE}$ & Centro-Oeste (MT) \\
\hline \multirow[t]{2}{*}{ PASSIFLORACEAE } & Turnera diffusa Willd. ex Schult. & CAA, CE, MA & Nordeste (CE, RN, PB, PE, BA), Sudeste (MG) \\
\hline & Turnera subulata $\mathrm{Sm}$. & $\mathrm{AM}, \mathrm{CAA}, \mathrm{CE}, \mathrm{MA}$ & $\begin{array}{c}\text { Norte (AP, PA, AM, RO), Nordeste, Centro-Oeste (MT, GO, } \\
\text { DF, MS), Sudeste (ES, SP, RJ) }\end{array}$ \\
\hline PLANTAGINACEAE & Tetraulacium veroniciforme Turcz. & CAA, MA, PAT & $\begin{array}{l}\text { Nordeste (MA, PI, CE, PB, PE, BA, AL), } \\
\quad \text { Centro-Oeste (MS), Sudeste (RJ) }\end{array}$ \\
\hline
\end{tabular}


Tabela 1. Continuação.

\begin{tabular}{|c|c|c|c|}
\hline FAMÍLIA & ESPÉCIES & $\begin{array}{l}\text { DOMÍNIOS } \\
\text { FITOGEOGRÁFICOS } \\
\text { (Forzza et al., 2012) }\end{array}$ & $\begin{array}{l}\text { DISTRIBUIÇÃO GEOGRÁFICA } \\
\quad \text { (Forzza et al., 2012) }\end{array}$ \\
\hline \multirow[t]{13}{*}{ POACEAE } & Andropogon bicornis Forssk. & AM, CAA, CE, MA, PAM, PAT & $\begin{array}{l}\text { Norte (RR, AP, PA, AM, TO, AC, RO), Nordeste (MA, PI, } \\
\text { CE, PB, PE, BA, AL, SE), Centro-Oeste, Sudeste, Sul }\end{array}$ \\
\hline & ${ }^{A}$ Aristida laevis (Nees) Kunth & CE, MA, PAM & Centro-Oeste (MS), Sudeste (MG, SP), Sul \\
\hline & Axonopus capillaris (Lam.) Chase & $\mathrm{AM}, \mathrm{CAA}, \mathrm{CE}, \mathrm{MA}$ & Nordeste, Centro-Oeste (GO), Sudeste (MG, SP) \\
\hline & $\begin{array}{l}\text { Axonopus polydactylus } \\
\text { (Steud.) Dedecca }\end{array}$ & $\mathrm{AM}, \mathrm{CAA}, \mathrm{CE}, \mathrm{MA}$ & Nordeste, Sudeste (SP), Sul (PR, RS) \\
\hline & $\begin{array}{l}\text { Axonopus pressus } \\
\text { (Nees ex Steud.) Parodi }\end{array}$ & AM, CAA, CE, MA & $\begin{array}{l}\text { Nordeste, Centro-Oeste (MT, GO, DF, MS), } \\
\text { Sudeste (MG, SP), Sul (PR) }\end{array}$ \\
\hline & ${ }^{B}$ Chaetium festucoides Nees & CAA, MA & Nordeste (MA, CE, PE, SE) \\
\hline & $\begin{array}{l}\text { Chaetochloa poiretiana } \\
\text { (Schult.) Hitchc. }\end{array}$ & CE, MA & $\begin{array}{l}\text { Norte (RR, PA), Nordeste (CE, PB, PE, BA), } \\
\text { Centro-Oeste (MT, GO, DF), Sudeste (MG, SP, RJ), Sul }\end{array}$ \\
\hline & ${ }^{B}$ Chloris barbata Sw. & CAA & Nordeste (BA) \\
\hline & $\begin{array}{l}\text { Mesosetum loliiforme } \\
\text { (Hochst. ex Steud.) Chase }\end{array}$ & $\mathrm{CE}$ & $\begin{array}{l}\text { Norte (RR, AP, PA, AM, RO), Nordeste (MA, PI, CE, RN, } \\
\text { BA), Centro-Oeste, Sudeste (MG) }\end{array}$ \\
\hline & Olyra latifolia $\mathrm{L}$. & $\mathrm{CE}$ & $\begin{array}{l}\text { Norte (RR, AP, AM, RO), Nordeste (MA, CE, PB, PE, BA), } \\
\text { Centro-Oeste, Sudeste, Sul }\end{array}$ \\
\hline & $\begin{array}{l}\text { Paspalum carinatum } \\
\text { Humb. \& Bonpl. ex Fluggé }\end{array}$ & AM, CAA, CE, MA, PAT & $\begin{array}{l}\text { Norte (RR, AM, PA, TO), Nordeste (MA, RN, BA), Centro- } \\
\text { Oeste, Sudeste (MG, SP), Sul (PR, SC) }\end{array}$ \\
\hline & Paspalum gardnerianum Nees & $\mathrm{AM}, \mathrm{CAA}, \mathrm{CE}$ & $\begin{array}{l}\text { Norte (RR, AP, PA, AM, TO), Nordeste (MA, PI, RN, PB, } \\
\text { PE, BA), Centro-Oeste, Sudeste (MG, SP) }\end{array}$ \\
\hline & $\begin{array}{l}\text { Trachypogon spicatus (L. f.) } \\
\text { Kuntze }\end{array}$ & $\mathrm{AM}, \mathrm{CAA}, \mathrm{CE}, \mathrm{MA}$ & Nordeste (BA), Centro-Oeste, Sudeste (MG, SP) \\
\hline \multirow[t]{2}{*}{ POLYGALACEAE } & Polygala hebeclada DC. & $\mathrm{CE}$ & $\begin{array}{l}\text { Nordeste (BA), Centro-Oeste (MT, GO, DF), } \\
\text { Sudeste (MG, SP, RJ), Sul (PR, SC) }\end{array}$ \\
\hline & Polygala longicaulis Kunth & $\mathrm{AM}, \mathrm{CE}, \mathrm{MA}$ & $\begin{array}{l}\text { Norte (RO, AP, PA, AM, TO), Nordeste (PI, RN, PB, BA), } \\
\text { Centro-Oeste (MT, GO, DF, MS), Sudeste (MG, SP), Sul }\end{array}$ \\
\hline \multirow[t]{2}{*}{ POLYGONACEAE } & Coccoloba latifolia Lam. & $\mathrm{AM}, \mathrm{CAA}, \mathrm{CE}$ & $\begin{array}{l}\text { Norte (RR, AP, PA, AM, TO, AC), } \\
\quad \text { Nordeste (MA, PI, CE, RN) }\end{array}$ \\
\hline & Coccoloba ramosissima Wedd. & AM, MA, PAM, PAT & $\begin{array}{c}\text { Norte (PA), } \\
\text { Nordeste (MA, PI, CE, RN, PB, BA), } \\
\text { Sudeste (ES, RJ) }\end{array}$ \\
\hline \multirow[t]{6}{*}{ RUBIACEAE } & ${ }^{B}$ Alseis pickelli Pilger \& Schmale & CAA, MA & Nordeste (CE, RN, PB, PE, BA, AL) \\
\hline & $\begin{array}{l}\text { Borreria verticillata (L.) G.F.W. } \\
\text { Meyer }\end{array}$ & $\mathrm{AM}, \mathrm{CAA}, \mathrm{CE}$ & $\begin{array}{l}\text { Norte (RR, PA, AM, RO), } \\
\text { Nordeste (MA, PI, CE, RN, PB, PE, BA), } \\
\text { Centro-Oeste (MT, GO, DF), Sudeste, Sul }\end{array}$ \\
\hline & Chiococca alba (L.) Hitchc. & $\mathrm{AM}, \mathrm{CAA}, \mathrm{CE}, \mathrm{MA}$ & $\begin{array}{l}\text { Norte (AC), Nordeste (RN, PB, PE, BA, AL, SE), } \\
\text { Centro-Oeste (MT, MS), Sudeste, Sul (PR) }\end{array}$ \\
\hline & Chiococca nitida Benth. & AM, MA & $\begin{array}{l}\text { Norte (PA, AM), } \\
\text { Nordeste (CE, RN, PB, BA, AL, SE), } \\
\text { Sudeste (ES) }\end{array}$ \\
\hline & $\begin{array}{l}{ }^{B} \text { Guettarda angelica } \\
\text { Mart. ex Müll.Arg }\end{array}$ & CAA & Nordeste (CE, PB, PE, BA) \\
\hline & Tocoyena bullata (Vell.) Mart. & CAA, CE, MA & $\begin{array}{l}\text { Nordeste (PB, BA, SE), } \\
\text { Sudeste (ES, SP, RJ) }\end{array}$ \\
\hline SALICACEAE & Casearia sylvestris $\mathrm{Sw}$. & AM, CAA, CE, MA, PAM, PAT & Norte, Nordeste, Centro-Oeste, Sudeste, Sul \\
\hline SAPINDACEAE & $\begin{array}{l}\text { Allophylus edulis (A. St.-Hil., } \\
\text { Cambess. \& A. Juss.) Radlk. }\end{array}$ & $\mathrm{AM}$ & Norte (AC), Nordeste, Sul \\
\hline SIMAROUBACEAE & Simaba cedron Planch. & $\mathrm{AM}, \mathrm{CAA}, \mathrm{CE}, \mathrm{MA}$ & $\begin{array}{c}\text { Norte (RR, AP, PA, AM, TO, AC, RO), } \\
\text { Nordeste (MA, PI, CE, PB, PE, BA), } \\
\text { Centro-Oeste (MT, GO), Sudeste (ES) }\end{array}$ \\
\hline VIOLACEAE & Hybanthus calceolaria (L.) Oken & AM, CAA, CE, MA, PAT & $\begin{array}{l}\text { Norte (RR, PA, AM, TO), Nordeste, } \\
\text { Centro-Oeste (MT, DF, MS), Sudeste }\end{array}$ \\
\hline
\end{tabular}


que Casearia sylvestris foi considerada uma espécie generalista com uma ampla distribuição geográfica que coloniza a partir da Floresta Atlântica para as comunidades de Cerrado do interior (Oliveira-Filho \& Fontes 2000; Oliveira-Filho 2009; Garcia et al. 2011).

Quanto à afinidade com a Floresta Amazônica, esta possivelmente está relacionada à biogeografia do Bioma Savana, mais precisamente, o Cerrado. Bigarella et al. (1975) indicaram que as florestas Amazônica e Atlântica teriam conexões ocorridas através do Brasil Central ("ponte Sudeste-Noroeste"), seja na forma de várias manchas florestais, por meio das quais as espécies poderiam ter "saltado ilhas", como de um corredor florestal. Dentro deste contexto, as florestas do Brasil Central constituem um importante elo entre a vegetação do nordeste, sudeste, Pantanal e formações sazonais e pré-amazônica (Oliveira-Filho \& Ratter 2000; Haidar et al. 2005) e a sua composição florística, naturalmente, é influenciada por estas zonas (Leitão Filho 1987). Além disso, a dispersão e a reciprocidade de espécies entre biotas são dependentes de configurações geográficas e eventos históricos (Schluter \& Ricklefs 1993).

\section{Novos registros, espécies ameaçadas e endêmicas}

Os dados obtidos indicam números significativos principalmente de novas ocorrências para o estado, correspondendo a $42,5 \%$ do total das espécies registradas em Rio do Fogo. Uma possível explicação para este fato deve-se: 1) a deficiência de coletas no referido estado tanto por especialistas locais como por estudiosos de outros estados e 2) que o estado conta ainda com poucos especialistas com atuação nas áreas de taxonomia e florística. Consequentemente, as coleções do Rio Grande do Norte são pouco citadas na literatura. Destaca-se que com esse estudo, a área de distribuição de Aristida laevis (Poaceae) foi ampliada.

Aspilia procumbens (Asteraceae) merece nossa atenção, uma vez que segundo Nakajima \& Mondin (2012) foi registrada apenas no semiárido potiguar e que esta região vem sofrendo ao longo do tempo, as mais diversificadas intervenções antrópicas.

Considerando as informações apresentadas, podemos destacar o potencial da área para conservação quando enfatizamos o lado positivo das novas citações indicadas no presente estudo, tanto para o estado quanto para a região. Quando comparamos a lista de espécies de Rio do Fogo com a investigação realizada durante seis anos (2001-2007) em São Paulo $\left(248.209,426 \mathrm{~km}^{2}\right)$, que resultou na "Flora Fanerogâmica do estado de São Paulo" é possível verificar que das 7500-8000 espécies vegetais (Mamede 2003; Shepherd 2003) estimadas para o estado, 61 espécies foram consideradas como desconhecidas e 156 novos registros para esse estado (Wanderley et al. 2001, 2002, 2003, 2005, 2007).

Com base na lista das espécies registradas e nas discussões apresentadas, foi possível averiguar que a área descrita somente por imagens de radar (RADAMBRASIL 1976) pode ser considerada como pertencente ao domínio do Cerrado. Assim, acredita-se que esse estudo contribuiu para ampliação do conhecimento da flora potiguar, bem como, da região Nordeste, apresentando dados robustos para a conservação desta área.

Por outro lado, os resultados obtidos poderão fornecer informações para atender aos objetivos fixados pela Estratégia Global para a Conservação das Plantas e da Convenção sobre a Diversidade Biológica através da expansão global de coleções botânicas, no Rio Grande do Norte, um dos estados menos amostrados do Brasil.

\section{Agradecimentos}

As autoras agradecem aos curadores dos herbários UFRN, ESA, UB e IBGE; a todos os especialistas que auxiliaram na identificação do material botânico; à Profa. Dra. Helena Freitas (Universidade de Coimbra) e Dra. Natália M. Ivanauskas (Instituto Florestal/SP) pelas críticas e sugestões do manuscrito; à Fundação Grupo Boticário de Proteção à Natureza e Fundação de Apoio à Pesquisa do Estado do Rio Grande do Norte (FAPERN) pelo financiamento parcial desta pesquisa e ao Programa REUNI/CAPES pela bolsa de mestrado concedida a A.C.P. de Oliveira.

\section{Referências bibliográficas}

Andrade, L.A.Z.; Felfili, J.M. \& Violatti, L. 2002. Fitossociologia de uma área de cerrado denso na RECOR-IBGE, Brasília-DF. Acta Botanica Brasilica 16(2): 225-240.

Andrade, M.C. de. 1967. Condições naturais do nordeste. Boletim Geográfico 19: 3-29.

APG [= Angiosperm Phylogeny Group] III. 2009. An update of the Angiosperm Phylogeny Group classification for the orders and families of flowering plants: APG III. Botanical Journal of the Linnean Society 161(2): 105-121.

Balduino, A.P.C.; Souza, A.L.; Meira Neto, J.A.A.; Silva, A.F. \& Silva Junior, M.C. 2005. Fitossociologia e análise comparativa da composição florística do cerrado da flora de Paraopeba - MG. Revista Árvore 29(1): 25-34.

Barbosa, M.R.V.; Mayo, S.J.; Castro, A.A.J.F.; Freitas, G.L.; Pereira, M.S.; Gadelha Neto, P.C. \& Moreira, H.M. 1996. Checklist preliminar das angiospermas. Pp. 253-415. In: Sampaio, E.V.S.B.; Mayo, S.J. \& Barbosa, M.R. (Eds.). Pesquisa Botânica Nordestina: Progresso e Perspectivas. Recife, Sociedade Botânica do Brasil, Seção Regional de Pernambuco.

Batalha, M.A. \& Mantovani, W. 2000. Reproductive phenological patterns of cerrado plant species at the Pé-de-Gigante Reserve (Santa Rita do Passa Quatro, SP, Brasil): a comparation between the herbaceous and woody floras. Revista Brasileira de Biologia 60(1): 129-145.

Bigarella, J.J.; Andrande, D. \& Riehs, P.J. 1975. Considerações a Respeito das Mudanças Paleoambientais na Distribuição de algumas Espécies Vegetais e Animais no Brasil. Pp. 411-464. In: Simpósio Internacional sobre o Quartenário, v. 47, Curitiba.

Biodiversitas. 2008. Lista oficial de espécies ameaçadas de extinção no Brasil. Disponível em http://www.biodiversitas.org.br./florabr/ grupo3fim.asp. (Acesso em: 13/11/2010).

Brown, J.H. \& Gibson, A.C. 1983. Distribution patterns of plants. Biogeography. St. Louis, C.V. Mostely. 375-411.

Carvalho G.H.; Cianciaruso M.V. \& Batalha M.A. 2010. Plantminer: a web tool for checking and gathering plant species taxonomic information. Environmental Modelling \& Software 25: 815-816. 
Cerqueira, R. 2000. Biogeografia das restingas. Pp. 65-76. In: Esteves, F.A. \& Lacerda, L.D. (Eds.) Ecologia de Restingas e Lagoas Costeiras. Macaé, UFRJ / NUPEM,

Cestaro, L.A. \& Soares, J.J. 2008. The Arboreal Layer of a Lowland Semideciduous (Tabuleiro) Forest Fragment in Rio Grande do Norte, Brazil. Memoirs of the New York Botanical Garden 100: 417-438.

Costa, A.A. \& Araújo, G.M. 2001. Comparação da vegetação arbórea de cerradão e de cerrado na Reserva do Panga, Uberlândia, Minas Gerais. Acta Botanica Brasilica 15(1): 63-72.

Costa, I.R.; Araújo, F.S. \& Lima-Verde, L.W. 2004. Flora e aspectos autoecológicos de um encrave de cerrado na chapada do Araripe, Nordeste do Brasil. Acta Botanica Brasilica 18(4): 759-770.

Durigan, G.; Franco, G.A.D.C.; Pastore, J.A. \& Aguiar, O.T. 1997. Regeneração natural da vegetação de Cerrado sob floresta de Eucalyptus citrodora. Revista do Instituto Florestal 9(1): 71-85.

Eiten, G. 1994. Vegetação do Cerrado. Pp. 1:9-65. In: Pinto, M.N. (Org.). Cerrado: caracterização, ocupação e perspectivas. Brasília: Editora Universidade de Brasilia.

Eiten, G. 1972. The cerrado vegetation of Brazil. The Botanical Review 38: 201-341.

Felfili, J.M. \& Silva Jr., M.C. 1993. A comparative study of cerrado (sensu stricto) vegetation in Central Brazil. Journal of Tropical Ecology 9: 277-289.

Felfili, J.M.; Filgueiras, T.S.; Haridasan, M.; Silva Júnior, M.C.; Mendonça, R. \& Rezende, A.V. 1994. Projeto biogeografia do bioma cerrado: Vegetação e solos. Caderno de Geociências do IBGE 12: 75-166.

Filgueiras, T. \& Pereira, B.A.S. 1990. Flora do Distrito Federal. Pp. 331388. In: Pinto, M.N. (Org.). Cerrado: caracterização, ocupação e perspectivas. Brasília, EUnB/SEMATEC.

Filgueiras, T. 2002. Herbaceous plant communities. Pp. 121-139. In: Oliveira, O.S. \& Marquis, R.J. (Eds.). The Cerrados of Brazil: ecology and natural history of a neotropical savanna. New York, Columbia University Press.

Filgueiras, T.S.; Felfili, J.M.; Silva-Junior, M.C.; Nogueira, P.E. 1998. Floristic and structural comparison of cerrado (sensu stricto) vegetation in Central Brazil. Pp. 633-647. In: F. Dallmeier; J Comiskey, A. \& Washington, D.C. (Eds.). Forest biodiversity in North, Central and South America and the Caribbean: research and monitoring. v. 21. Carnforth, The Parthenon Publishing Group.

Forzza, R.C.; Leitman, P.M.; Costa, A.F.; Carvalho Jr., A.A.; Peixoto, A.L.; Walter, B.M.T.; Bicudo, C.; Zappi, D.; Costa, D.P.; Lleras, E.; Martinelli, G.; Lima, H.C.; Prado, J.; Stehmann, J.R.; Baumgratz, J.F.A.; Pirani, J.R.; Sylvestre, L.; Maia, L.C.; Lohmann, L.G.; Queiroz, L.P.; Silveira, M.; Coelho, M.N.; Mamede, M.C.; Bastos, M.N.C.; Morim, M.P.; Barbosa, M..; Menezes, M.; Hopkins, M.; Secco, R.; Cavalcanti, T. B. \& Souza, V.C. 2012. Introdução. In: Lista de Espécies da Flora do Brasil. Jardim Botânico do Rio de Janeiro. Disponível em http://floradobrasil.jbrj. gov.br/2012/ (Acesso em 23/03/2012).

Garcia, P.O.; Valente, A.S.M.; Pifano, D.S.; Pessoa, J.F.S.; Busato, L.C.; Fontes, M.A.L. \& Oliveira-Filho, A.T. Species composition and floristic relationships in southern Goiás forest enclaves. Rodriguesia 62(1): 123-137. 2011.

Giulietti, A.M.; Rapini, A.; Andrade, M.J.G.; Queiroz, L.P. \& Silva, J.M.C. 2009. Plantas raras do Brasil. Belo Horizonte, Conservação Internacional.

Goodland, R. 1979. Análise ecológica da vegetação de cerrado. Pp. 61-179. In: Goodland, R. \& Ferri, M.G. Ferri. (Eds.). Ecologia do cerrado. São Paulo, EDUSP

Haidar, R.F; Felfili, J.M.; Fagg, C.W. \& Pinto, J.R.R. 2005. Fitossociologia da vegetação arbórea em fragmentos de floresta estacional, Parque Nacional Ecológico Altamiro de Moura Pacheco, GO. Boletim Herbário Ezchias Paulo Heringer 15: 19-46.

Heringer, E.P.; Barroso, G. M.; Rizzo, I. A. and Rizzini, C. T. (1977), A flora do cerrado. In: Ferri, M. G. (Coord.). Simpósio sobre o cerrado: bases para a utilização agropecuária. São Paulo: EDUSP. pp. 211-232.

Heringer, E.P, Barroso, G.M, Rizzo, J.A. \& Rizzini, C.T. 1977. A flora do Cerrado. Pp. 211-232. In.. Ferri M.G. (Coord.). IV Simpósio Sobre o Cerrado. v. 4. São Paulo, EDUSP.
Instituto de Desenvolvimento Econômico e do Meio Ambiente (IDEMA). 2002. Perfil do Estado do Rio Grande do Norte. Natal, IDEMA.

Kellogg, E.A. 1998. Relationships of cereal crops and other grasses. Proceedings of the National Academy of Sciences 95: 2005-2010.

Kellogg, E.A. 2001. Evolutinary history of the grasses. Plant Physiology 125: 1198-1205.

Köppen, W.P. 1948. Climatologia: com um estúdio de los climas de la tierra. México, Fondo de Cultura Econômica.

Leitão Filho, H.F. 1987. Considerações sobre a florística de florestas tropicais e sub-tropicais do Brasil. Instituto de Pesquisas e Estudos Florestais 35: 41-46.

Mamede, M.C.H. 2003. A experiência do projeto Flora Fanerogâmica do Estado de São Paulo. Pp. 127-139. In: Peixoto, A.L. (Ed.). Coleções biológicas de apoio ao inventário, uso sustentável e conservação da biodiversidade. Rio de Janeiro, Instituto de Pesquisas Jardim Botânico do Rio de Janeiro.

Mantovani, W. \& Martins, F.R. 1993. Florística do Cerrado na Reserva Biológica de Moji Guaçu, SP. Acta Botanica Brasilica 7(1): 33-60.

Martins, F.R. 1993. Estrutura de uma floresta mesófila. $2^{\text {a }}$ ed. Campinas, Editora da Unicamp.

Méio, B.B.; Freitas, C.V.; Jatobá, L.; Silva, M.E.F.; Ribeiro, J.F. \& Henriques, R.P.B. 2003. Influência da flora das florestas Amazônicas e Atlânticas na vegetação do cerrado sensu stricto. Revista Brasileira de Botânica 26(4): 437-444.

Mendonça, R.C. 1998. Flora vascular do cerrado. Pp. 290-556. In: Sano, S.M \& Almeida, S.P. (Eds.). Cerrado: ambiente e flora. Planaltina, EMBRAPA/CPAC.

MMA - Ministério do Meio Ambiente. Instrução Normativa $\mathrm{n}^{\circ}$ 6, de 23 de setembro de 2008. Disponível em http://www.mma.gov.br/estruturas/179/_arquivos/179_05122008033615.pdf. (Acesso em 23/06/2009).

MOBOT. 2008. Missouri Botanical Garden, $W^{3}$ Specimen Data Base. Disponível em http://www.mobot.org/plantscience/W3T/Search/vas. html. (Acesso em: 09/01/2010).

Mori, S.A., Silva, L.A.M., Lisboa, G.; Coradin, L. 1989. Manual de manejo do herbário fanerogâmico. $2^{\text {a }}$ ed. Ilhéus, Centro de Pesquisas do Cacau.

Munhoz, C.B.R. \& Felfili, J.M. 2006. Floristics of the herbaceous and subshrub layer of a moist grassland in the Cerrado Biosphere Reserve (Alto Paraíso de Goiás), Brazil. Edinburgh Journal of Botany 63(2): 343-354.

Nakajima, C.A. \& Mondin, J. 2012. Aspilia. In: Forzza et al. (Orgs.). Lista de Espécies da Flora do Brasil. Jardim Botânico do Rio de Janeiro. Disponível em http://floradobrasil.jbrj.gov.br/2010/FB026834. (Acesso em $25 / 03 / 2012$ ).

Oliveira-Filho, A.T. \& M.A.L. Fontes. 2000. Patterns of floristic differentiation among Atlantic Forests in southeastern Brazil and the influence of climate. Biotropica 32(4b): 793-810.

Oliveira-Filho, A.T. \& Ratter, J.A. 2000. Padrões florísticos das matas ciliares da região dos cerrados e a evolução das paisagens do Brasil central durante o Quaternário tardio. Pp. 73-89. In: Rodrigues, R.R. \& Leitão Filho, H.F. (Eds.). Matas ciliares: bases multidisciplinares para estudo, conservação e restauração. São Paulo, EDUSP.

Oliveira-Filho, A.T. 2009. Treeatlan 1.0: Flora arbórea da Mata Atlântica e domínios adjacentes - um banco de dados envolvendo geografia, diversidade e conservação. Disponível em http://http://www.icb. ufmg. br/treeatlan. (Acesso 07/12/2010).

Peixoto, A.L. \& Thomas, W.W. 2005. Listas florísticas, floras regionais e flora do Brasil: desafios e estratégias. Disponível em http//:www.cria. org.br/cgee/col. (Acesso 13/11/2010).

Pereira, O.J. \& Araújo, D.S.D. 2000. Análise florística das restingas dos Estados do Espírito Santo e Rio de Janeiro. Pp. 25-63. In: Esteves, F.A. \& Lacerda, L.D. (Eds.). Ecologia de Restingas e Lagoas Costeiras. Macaé, UFRJ / NUPEM.

Radambrasil. Departamento Nacional de Produção Mineral. 1976. Folha AS.21-. Geologia, geomorfologia, pedologia, vegetação e uso potencial da terra. Levantamento dos Recursos Naturais. v. 10. Rio de Janeiro: DNPM.

Ratter, J.A.; Ribeiro, J.F.L. \& Bridgewater, S. Brazilian cerrado vegetation and threats to its biodiversity. 1997. Annals of Botany 80: 223-230. 
Ribeiro, J.F. \& Walter, B.M.T. 1998. Fitofisionomias do Bioma Cerrado. Pp.89-166. In: sano, S.M. \& Almeida, S.P. (Eds.). Cerrado: ambiente e flora. Planaltina, EMBRAPA.

Rizzini, C.T. 1963. Nota prévia sobre a divisão fitogeográfica (florísticosociológica) do Brasil. Revista Brasileira de Geografia 25: 3-65.

Rizzini, C.T. 1997. Tratado de fitogeografia do Brasil: Aspectos e Ecológicos, Sociológicos e Florísticos. Rio de Janeiro, Editora Âmbito Cultural.

Scarano, F.R.; Cirne, P.; Nascimento, M.T.; Sampaio, M.C.; Villela, D.M.; Wendt, T. \& Zaluar, H.L.T. 2004. Ecologia vegetal: integrando ecossistemas, comunidades, populações e organismos. Pp. 77-97. In: Rocha, C.F.D.; Esteves, F.A. \& Scarano, F.R. (Eds.). Pesquisas de longa duração na restinga de Juruabatiba. Ecologia, história natural e conservação. São Carlos, Rima Editora.

Schluter, D. \& Ricklefs, R.E. 1993. Species diversity: regional and historical influences. Pp. 350-364. In: Ricklefs, R.E. \& Schuter, D. (Eds.). Species diversity in ecological communities. Chicago, University of Chicago.

Shepherd, G.J. 2003. Avaliação do estado do conhecimento da diversidade biológica do Brasil: plantas terrestres (versão preliminar). Ministério do Meio Ambiente, Brasília. Disponível em http//: www.mma.gov. br/estruturas/chm/_arquivos/plantas1.pdf (Acesso em 07/11/2010).

Silva, M.A. \& Nogueira, P.E. 1999. Avaliação fitossociológica do estrato arbustivo-herbáceo em cerrado stricto sensu após incêndio acidental, no Distrito Federal, Brasil. Boletim do Herbário Ezechias Paulo Heringer 4: 65-78.
Tabarelli, M. \& Vicente, A. 2004. Conhecimento sobre plantas lenhosas da Caatinga: lacunas geográficas e ecológicas. Pp. 101-110. In: Silva, J.M.C.; Tabarelli, M., Fonseca, M. \& Lins, L. (Eds.). Biodiversidade da Caatinga: áreas e ações prioritárias para a conservação. Brasília, Ministério do Meio Ambiente.

Thiers, B. 2012. Index Herbariorum: a global directory of public herbaria and associated staff. New York Botanical Gardens Virtual Herbarium. Disponível em http://sweetgum.nybg.org/ih (Acesso em 24/03/2012).

Vargas, M.A.T. \& Hungria, M. 1997. Biologia dos solos dos Cerrados. Brasília, EMBRAPA-CPAC.

Wanderley, M.G.L.; Shepherd, G.J.; Giulietti, A.M. \& Melhem, T.S. 2003. Flora Fanerogâmica do Estado de São Paulo 3: 367.

Wanderley, M.G.L.; Shepherd, G.J.; Giulietti, A.M. 2001. Flora Fanerogâmica do Estado de São Paulo 1: 292.

Wanderley, M.G.L.; Shepherd, G.J.; Giulietti, A.M. 2002. Flora Fanerogâmica do Estado de São Paulo 2: 31.

Wanderley, M.G.L.; Shepherd, G.J.; Melhem, T.S. \& Giulietti, A.M. 2005. Flora Fanerogâmica do Estado de São Paulo 4: 392

Wanderley, M.G.L.; Shepherd, G.J.; Melhem, T.S. \& Giulietti, A.M. 2007. Flora Fanerogâmica do Estado de São Paulo 5: 476.

Weiser, V.L. \& Godoy, S.A.P. 2001. Florística em um hectare de cerrado sensu stricto na ARIE - Cerrado Pé-de-Gigante, Santa Rita do Passa Quatro, SP. Acta Botanica Brasilica 15(2): 201-212. 